\title{
Mixing enhancement using chevron nozzle: studies on free jets and confined jets
}

\author{
G HARISH SUBRAMANIAN, CH V S NAGARJUN, K V SATISH KUMAR, B ASHISH KUMAR, \\ VISHAL SRIKANTH and A R SRIKRISHNAN*D
}

\author{
Department of Aerospace Engineering, Amrita School of Engineering, Amrita Vishwa Vidyapeetham, \\ Coimbatore, India \\ e-mail: ar_srikrishnan@cb.amrita.edu
}

MS received 31 January 2017; revised 6 March 2018; accepted 22 March 2018; published online 22 June 2018

\begin{abstract}
This paper reports an experimental study focused on the impact of chevrons (serrations on the trailing edge of the nozzle) on the mixing process of an incompressible jet issuing from a convergent nozzle. The study also explores enhancement of the mixing performance by a novel approach to geometry modification. Profiles of mean velocity were used to characterize the extent of mixing. For a comparative assessment, studies were carried out with a base line circular nozzle, a conventional chevron nozzle and an improvised tabbed chevron nozzle. Flow visualization studies were carried out for jets issuing from chevron nozzles and the results corroborate well with quantitative measurements. The impact of confinement on mixing of jets issuing from chevron nozzles is also studied. The results show that the proposed geometry modification can significantly improve the rate of mixing in the range of Reynolds numbers considered in the study. In confined jets, presence of chevrons was found to accelerate the process of jet break-down.
\end{abstract}

Keywords. Chevron nozzle; free jets; mixing enhancement; confined jets.

\section{Introduction}

Mixing enhancement of free jets finds widespread engineering applications, including noise reduction in aircraft gas turbine engines, fuel-air mixing in gas turbine combustors and pollutant spread in industries [1]. Since free jets entrain large amounts of surrounding fluid, they find wide application in industrial mixing systems (mixing in large tanks, stack exhaust mixing, etc.) [2]. Studies of Bernal and Roshko [3] and Liepmann and Gharib [4] on plane and axisymmetric mixing layer revealed the existence of orderly streamwise and spanwise structures. They reported formation of $\mathrm{K}-\mathrm{H}$ (Kelvin-Helmholtz) and streamwise structures; while the K$\mathrm{H}$ structures pair up and diminish downstream, the streamwise structures are long-lasting and are more efficient in entrainment. Once the importance of the streamwise vortices in governing the entrainment and mixing of an axisymmetric free jet was established, introduction of additional streamwise vorticity into the jet by means of tabs, lobed nozzles and non-circular nozzles was explored by various studies. The physics of jet mixing with tabs has been extensively studied by Zaman et al [5] and Reeder and Samimy [6]. They observed that the tabs distort the cross section of the jet and introduce streamwise vortices, which enhance mixing. Each

*For correspondence tab introduces a pair of strong streamwise vortices, which bifurcates the jet as well as a pair of weaker horseshoe vortices at the periphery of the jet. Verma and Rathakrishnan [7] undertook acoustic and pressure measurements (using a Pitot probe) on plain and notched elliptic slot jets. The nozzle aspect ratio was found to be an important parameter in governing the mixing characteristics. Arun Kumar and Rathakrishnan [8] carried out pressure measurements for supersonic jets issuing from nozzles with sharp and truncated triangular tabs using a Pitot probe. The triangular tab was found to be a better mixing promoter compared with the rectangular tab. Srikrishnan et al $[9,10]$ have reported mixing enhancement studies using lobed nozzles in co-flowing supersonic jet streams. Nastase et al [11], Nastase and Meslem [12,13] and El Hassan and Meslem [14] carried out comprehensive flow visualization and PIV measurements on cross-orifice jets, daisy-shaped orifice jets and lobed jets. They observed that entrainment in these jets increases over that of circular jets because of introduced streamwise vorticity, unlike the case of circular jets, where the $\mathrm{K}-\mathrm{H}$ ring vortices suppress the growth of streamwise structures (which causes a decrease in instantaneous entrainment during the passing of a $\mathrm{K}-\mathrm{H}$ structure). In the cases of cross-shaped/ lobed/daisy jets the jet cross section is split into ring-shaped segments, which promotes the free development of streamwise structures. 


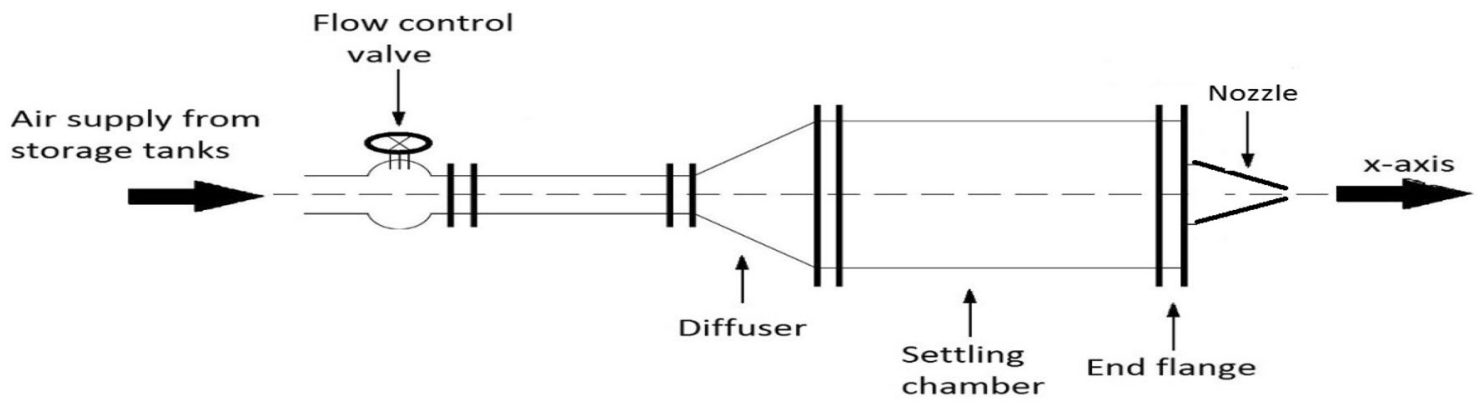

(a)

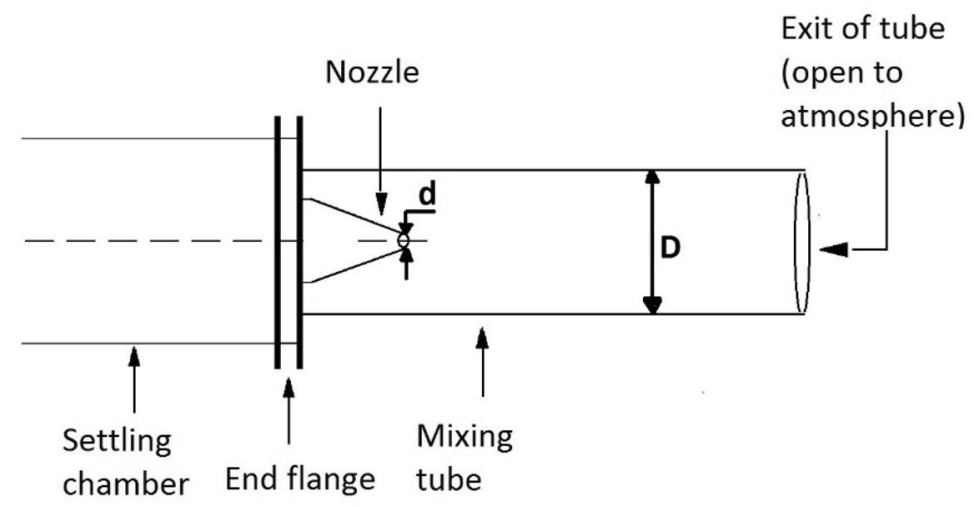

(b)

Figure 1. (a) Schematic of experimental set-up for free jets. (b) Schematic of the confined jet configuration.

Chevrons are serrations in the trailing edge of the nozzle, which induce vorticity into the shear layer [15] and hence enhance mixing between the high-speed jet and quiescent ambient. The key difference between tabs and chevrons is that geometric configuration of the latter provides for a more aggressive protrusion into the jet [16]. Several studies in the recent past have addressed the mixing enhancement of chevrons. Bridges and Brown [17] carried out parametric experimental analysis of single-flow hot jets, by varying chevron count, penetration and length. They observed that chevron length was not a significant factor in reducing the noise whereas the penetration and count strongly influenced the flow field. Callender et al [15] carried out PIV measurements on co-axial chevron jets. The study showed that momentum exchange in the transverse direction from the primary jet to secondary jet is one of the mechanisms by which chevrons increase mixing in co-axial jets. Another recent study using PIV measurements by Kennedy and Fitzpatrick [18] analysed the radial profiles of velocity along two characteristic planes for chevron nozzle. They reported that chevrons alter some of the fundamental properties of the jet - namely, potential core, shear layer thickness and turbulence intensity. They also deduced that chevron penetration has a significant effect on the potential core length. LES simulations of jets issuing from chevron and circular nozzles by Mihaescu and Gutmark [19], at Reynolds number around $10^{4}$, showed that the lobed structures generated by the chevron play an important role in the mixing process. It was also noticed that chevrons result in a faster decay in centreline velocity and a higher jet spread. The acoustic analysis reported in the study showed that chevrons induce strong lateral jets, which enhance mixing in the jet shear layer. Opalski et al [20] carried out PIV measurements on single-flow chevron nozzles in the Mach number range of 0.9-1.5. They observed the formation of counter-rotating structures in the notch between the adjacent teeth. The increase in jet-penetration was found to result in a decrease in the potential core length due to a significant increase in the axial or streamwise circulation.

The studies discussed earlier pertain to free-jet mixing (in the absence of a confinement). For circular nozzles, studies have been reported for confined jets as well. Risso and Fabre [21] carried out detailed experimental investigations on the confined circular jet flow. At some distance from the nozzle, a turbulent region was found to develop, where the kinetic energy of the mean flow almost vanishes. After a certain distance the jet loses its coherence and completely breaks down, having very low velocities. The decay of the centreline velocity was found to be 
independent of Reynolds number and the location where the centreline velocity goes to zero was independent of length of the tube as long as the tube was long enough. Also, the rate of decay of mean velocity was found to increase with decrease in tube diameter. Voropayev et al [22] observed a region of diffusive turbulence (region of very low jet velocities) after a certain critical distance. The critical break-down distance was found to be independent of Reynolds number. Liberzon and Fernando [23] showed experimentally that the presence of adverse pressure gradient was the main cause of jet break-up. Singh et al [24] carried out quantitative measurements using a Pitot probe for circular and non-circular confined jets. They observed that non-circular confined jets have greater mixing and entrainment compared with circular confined jets.

From previous studies it is now well established that chevrons increase mixing and entrainment in free jets. Also, formation of counter-rotating streamwise structures is identified as one of the primary factors in mixing enhancement. Parametric studies have revealed the impact of varying the tooth count, penetration and length of the tooth. However, relatively less data about chevron-induced mixing are available at lower Reynolds numbers (5000-9000 based on centreline velocity and nozzle exit diameter). Also, possibility of further enhancement of mixing by appropriate alterations to the geometry of chevrons needs to be explored due to the continued demand for faster mixing. There is also a paucity in research related to confined mixing of jets issuing from chevron nozzles while several studies have been reported for confined jets from circular nozzles.

The present study focuses on single jets issuing from chevrons at low Reynolds numbers. The following are the core issues that the study addresses in order to contribute to the body of knowledge in the domain:

I) The development of velocity profiles and the progress of mixing of a single incompressible jet issuing from chevron nozzle into stagnant atmosphere; comparison of mixing for this configuration to that for a circular nozzle.

II) Exploration of further enhancement of chevron-aided mixing by an improvised chevron geometry with tabs introduced at nozzle exit, referred to here as tabbed chevron.

III) Visualization of the jet cross section evolution so as to assess and compare development of the jets issuing from chevron and tabbed chevron nozzle.

IV) The impact of confinement on the mixing of jets issuing from chevrons - data are not available in open literature on confined mixing for chevron nozzles.

In the context of the present study, mixing is characterized by the jet spread and the decay in centreline velocity. A similar approach has been followed in several previous studies on mixing enhancement (e.g. $[7,9,10])$.

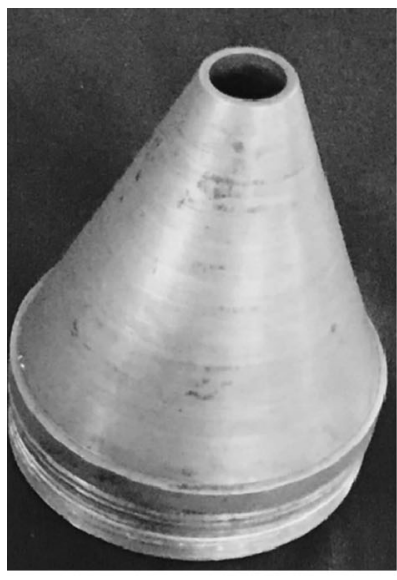

(a)

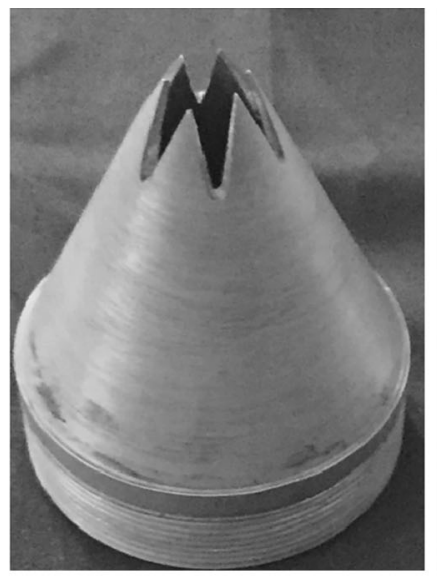

(b)

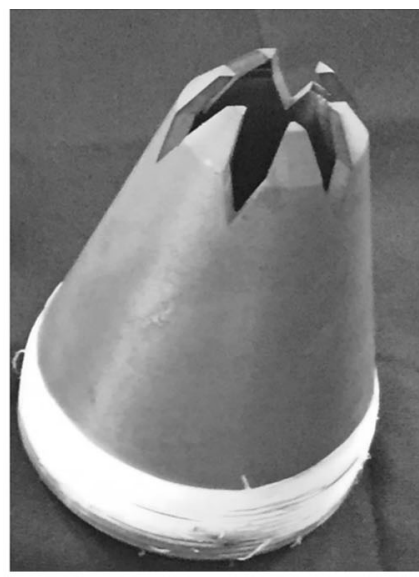

(c)

Figure 2. The nozzles used in the present study: (a) circular (BSL), (b) conventional chevron (ChN) and (c) improvised chevron with tabs $(\mathrm{T}-\mathrm{ChN})$.

Table 1. Salient dimensions of nozzles used in the present study.

\begin{tabular}{|c|c|c|c|}
\hline Dimension & $B S L$ & $C h N$ & $T-C h N$ \\
\hline Length of nozzle (mm) & 82.26 & 82.26 & 82.26 \\
\hline Exit diameter of nozzle $d(\mathrm{~mm})$ & 12 & 12 & 12 \\
\hline Inlet diameter of nozzle (mm) & 66 & 66 & 66 \\
\hline Semi-convergence angle (deg) & 17.25 & 17.25 & 13.85 \\
\hline Length of tooth $(\mathrm{mm})$ & - & 15 & 15 \\
\hline Bending angle of aft-half of the tooth with respect to inclined plane of the nozzle (deg) & - & 0 & 25.5 \\
\hline
\end{tabular}




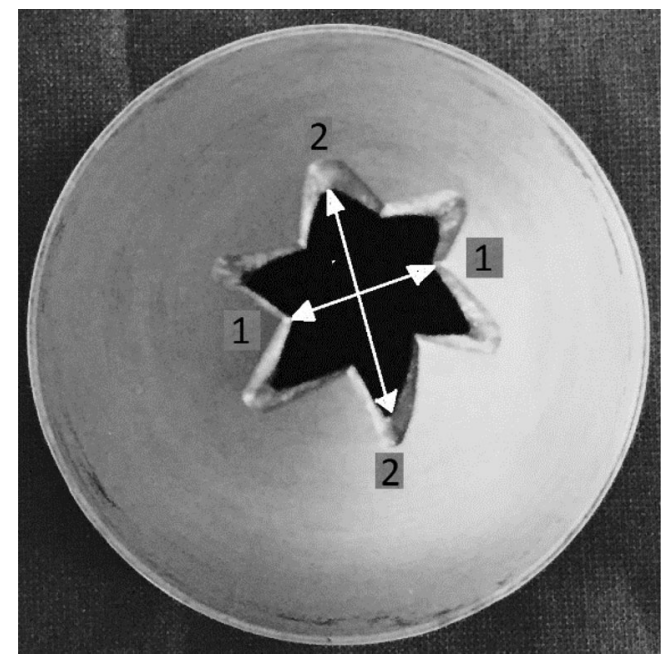

Figure 3. The minor and major planes of chevron nozzle: 1-1, minor plane; 2-2, major plane.

\section{Experimental set-up and procedure}

The incompressible free-jet facility used in the present study (shown schematically in figure 1) consists of a cylindrical settling chamber of diameter $135 \mathrm{~mm}$ connected to high-pressure storage tank by means of a diffuser. The settling chamber has provision to mount the nozzles on its end flange. Compressed dry air is supplied into the settling chamber through a pressure-regulating valve.

The study is primarily based on characterization of the development of the jet and its mixing with the stagnant atmosphere using profiles of mean velocity. Pressure is measured using a Pitot probe. Studies of Zaman et al [5], Singh et al [24] and Arun Kumar and Rathakrishnan [8] are examples of free-jet studies using Pitot probe measurements. The probe used in this study has an inner diameter of $0.4 \mathrm{~mm}$ and outer diameter of $0.6 \mathrm{~mm}$. The ratio of nozzle exit area to probe area is 400 ; this is well above the limit of

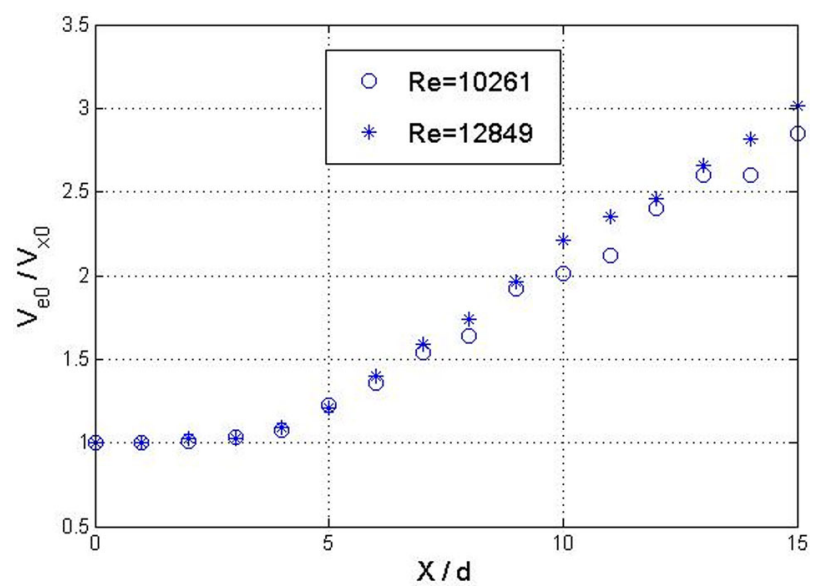

Figure 4. Variation of dimensionless inverse of centreline velocity with $X / d$ at different Reynolds numbers.
64 , which is the minimum value required for area ratio to neglect blockage effects [25, 26]. The velocity at the exit of the circular nozzle is nearly constant up to $83 \%$ of the nozzle diameter. The uncertainty in velocity measurements was estimated to be $4 \%$ (for both the free- and confined-jet cases). All velocity measurements reported in this paper were carried out using a Pitot probe mounted on a 2-dimensional traverse mechanism. The flow stagnation pressure was measured relative to the atmospheric pressure and the flow velocity at each point was then calculated using the well-known Bernoulli's equation, as the jet is well within the incompressible regime. The Reynolds numbers considered in the present study ranged from 6000 to 9000 for free-jet experiments.

In order to study the effect of confinement, a mixing tube was fixed rigidly to the end flange by means of a clamp as shown schematically in figure 1(b). The upstream end of the mixing tube was closed while the downstream end was open to the atmosphere. There was no secondary flow; hence the jet issues from the nozzle and expands in the mixing tube.

The wall static-pressure measurements for the confined jet case were carried out by drilling pressure ports on the walls of the confining tube and connecting them to a Multitube inclined U-Tube manometer, the experiments were carried out for two values of $D / d$ ratios: 5.25 and 6.25. The errors in pressure measurement were estimated to be $5 \%$. The velocity measurements for the confined jet and the free-jet cases were carried out using the pitot probe mentioned previously and the error in velocity measurements was estimated to be $4 \%$.

For flow visualization studies, incense smoke was introduced into the settling chamber. The jet cross sections were illuminated by a laser light sheet (this laser sheet was formed on dispersion of a laser source by a glass rod). For capturing the images, a CANON EOS-60D camera was used; the acquisition rate was $5.3 \mathrm{fps}$ and the shutter speed was maintained to be $1 / 10$.

\subsection{The nozzles used in the study}

In line with the objectives listed earlier, the present study was conducted with 3 nozzles: (i) a circular baseline nozzle (BSL nozzle), (ii) a six-tooth conventional chevron nozzle $(\mathrm{ChN})$ and (iii) an improvised six-tooth chevron nozzle with tabs provided at the exit section, referred to in this paper as the tabbed chevron nozzle (T-ChN). All the three nozzles (figure 2) are subsonic (convergent) and the pertinent dimensions are summarized in table 1 . The nozzles were made of brass.

As mentioned earlier, provision of the tab-like protrusion for the improvised geometry was conceptualized with the core objective of enhancing the mixing rate. Tabs have been used in the past in circular nozzle for mixing enhancement [5]. The tabs enhance mixing by introduction 
Table 2. Comparison of parameters from the present study with published data for circular jet.

\begin{tabular}{lccc}
\hline Parameter & Reference & Value reported in reference & Value in present study \\
\hline Virtual origin $(X 0 / d)$ & Hussein et al [27] & 5 & 4 \\
Jet decay constant $(A)$ & Abdel Rahman et al [28] & $6.06(\operatorname{Re}=11000)$ & $6.12(\operatorname{Re}=10261)$ \\
& Panchapakesan and Lumley [29] & $5.95(\operatorname{Re}=13200)$ & $5.93(\operatorname{Re}=12849)$ \\
Spread rate $(b)$ & Abdel Rahman et al [28] & $0.08(\operatorname{Re}=13200)$ & $0.078(\operatorname{Re}=12594)$ \\
Spread rate $(b)$ & Panchapakesan and Lumley [29] & $0.096(\operatorname{Re}=11000)$ & $0.11(\operatorname{Re}=10261)$ \\
\hline
\end{tabular}
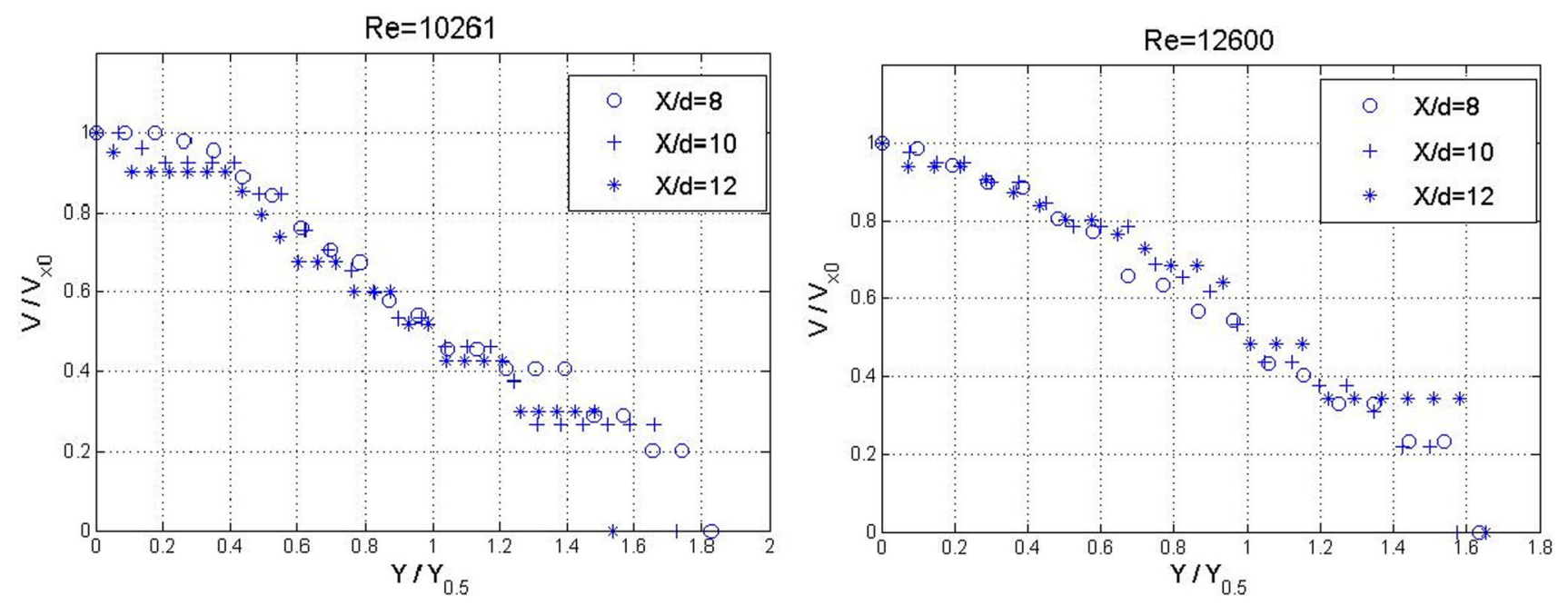

Figure 5. Radial profiles of mean velocity at different axial locations $(X / d)$, for BSL nozzle.

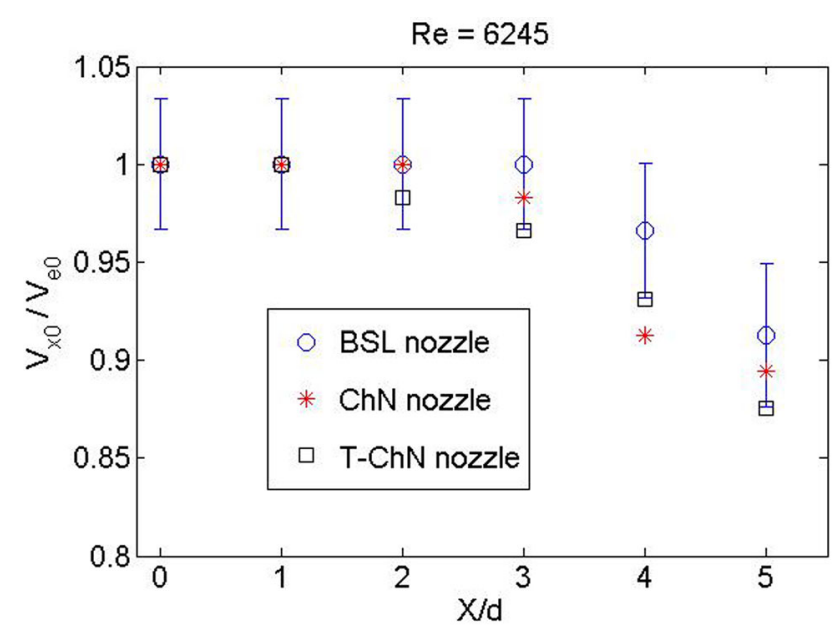

Figure 6. Decay of the centreline velocity for different nozzles (error bars shown are for the BSL data).

of streamwise vortices into the jet stream by way of a somewhat sharp projection. In the present study the feasibility of enhancing mixing further by a combination of chevrons and tabs is explored by way of designing the tabbed chevron nozzle. The tabbed chevron nozzle (T-ChN) was designed with the same length, exit diameter, tooth count and tooth length as those of the conventional chevron nozzle $(\mathrm{ChN})$ used in the study - for the tabbed chevron geometry, the aft-half of the tooth was bent inwards further by an angle of $25.5^{\circ}$ with respect to the inclined surface of the nozzle.

In order to characterize jet mixing in the radial as well as the azimuthal directions, two distinct planes were identified in the jets issuing from the chevron nozzles ( $\mathrm{ChN}$ and $\mathrm{T}-\mathrm{ChN})$. These planes are referred to as the major plane (along the outer tip of the chevron) and the minor plane (along the inner tip of the chevron). The two planes are marked in figure 3.

\section{Results and discussion}

The results of the present study are organized in 3 sections, based on the focus of the analysis, as follows:

1) benchmarking of the measurements by comparison with published experimental data,

2) studies on free-jet mixing enhancement with conventional and tabbed chevron nozzles and

3) studies on jet mixing with confinement. 
Table 3. Value of potential core length for different nozzles at different Reynolds numbers.

\begin{tabular}{lccc}
\hline Reynolds number & Circular nozzle (BSL) & Conventional chevron (ChN) & Tabbed chevron (T-ChN) \\
\hline 6245 & $3 d$ & $2 d$ & $1 d$ \\
7390 & $3 d$ & $2 d$ & $0 d$ \\
8900 & $2 d$ & $1 d$ & $0 d$ \\
\hline
\end{tabular}

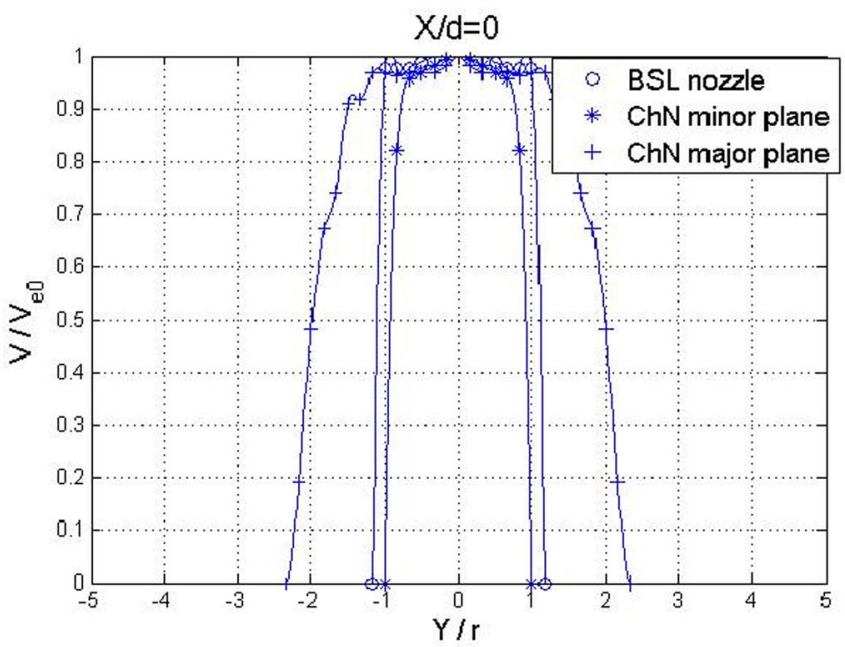

(a)

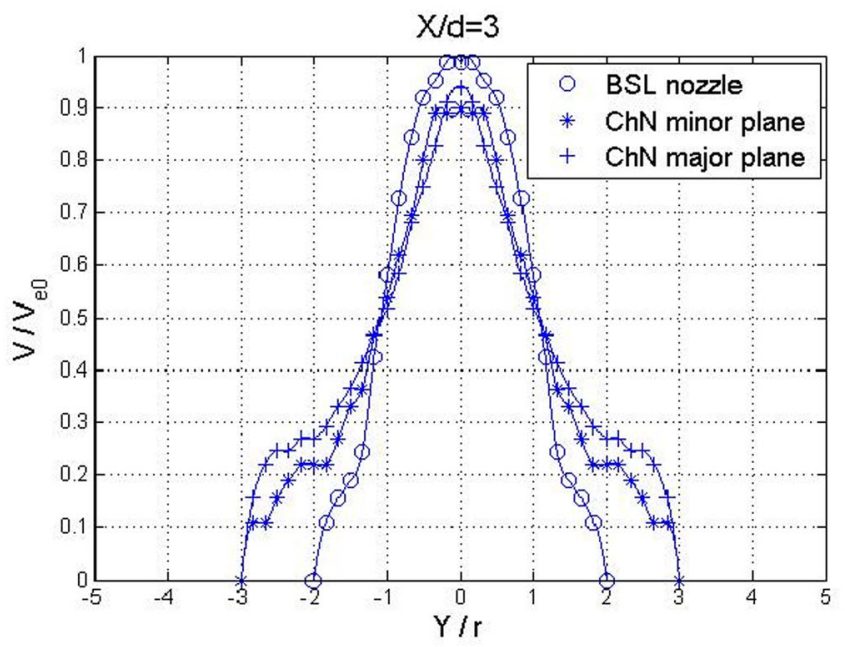

(c)

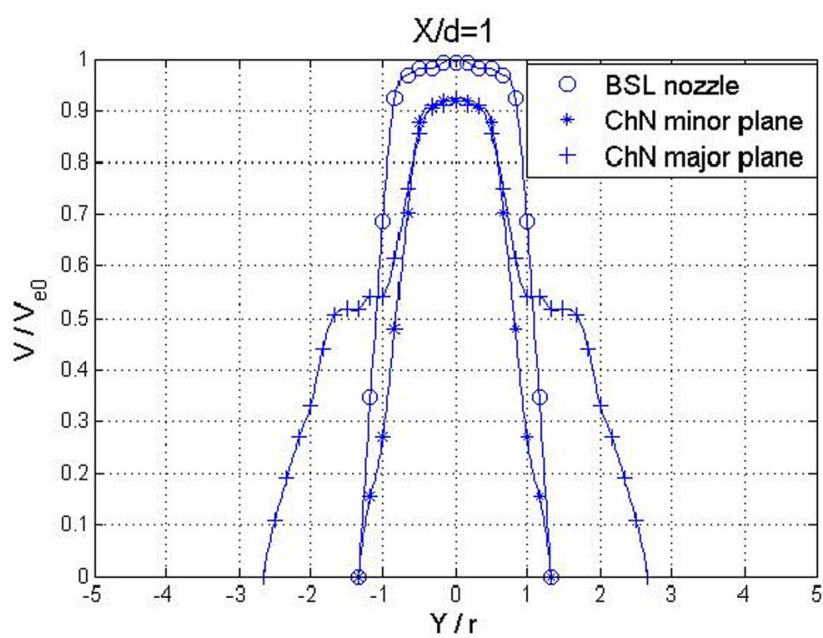

(b)

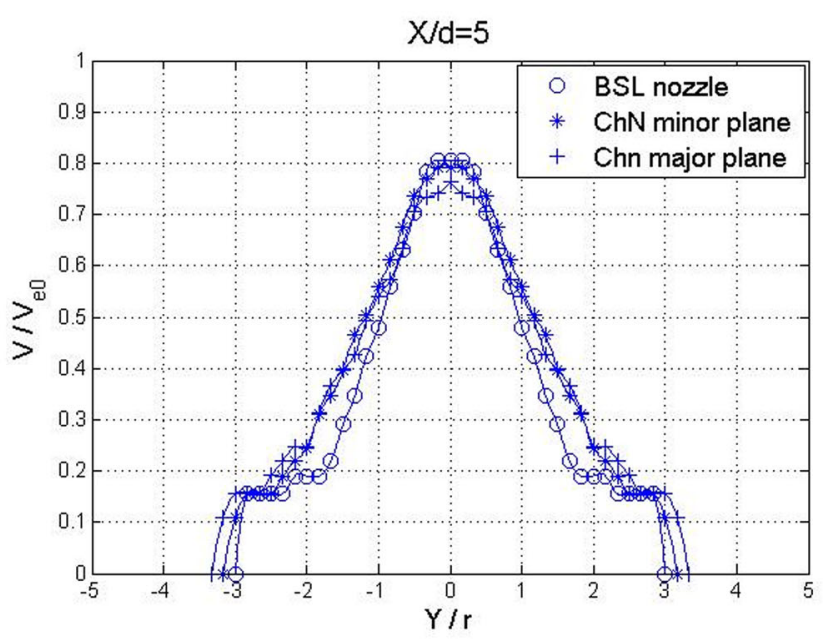

(d)

Figure 7. Mean axial velocity profiles in the radial direction for a Reynolds number of 10390 for baseline and conventional chevron nozzles.

\subsection{Comparison of baseline nozzle measurements with published data}

In order to benchmark the measurements made in the present study with published data, certain characteristic features of the jet (used commonly in jet flow research) were validated against those available in literature. The results discussed in this section pertain to the baseline circular nozzle.

Variation of dimensionless inverse of centreline velocity $\left(\frac{V_{e 0}}{V_{x 0}}\right)$ along axial direction is plotted in figure 4. This parameter remains constant till $X / d=4$ and then increases linearly. Virtual origin is defined [27] as the location up to 


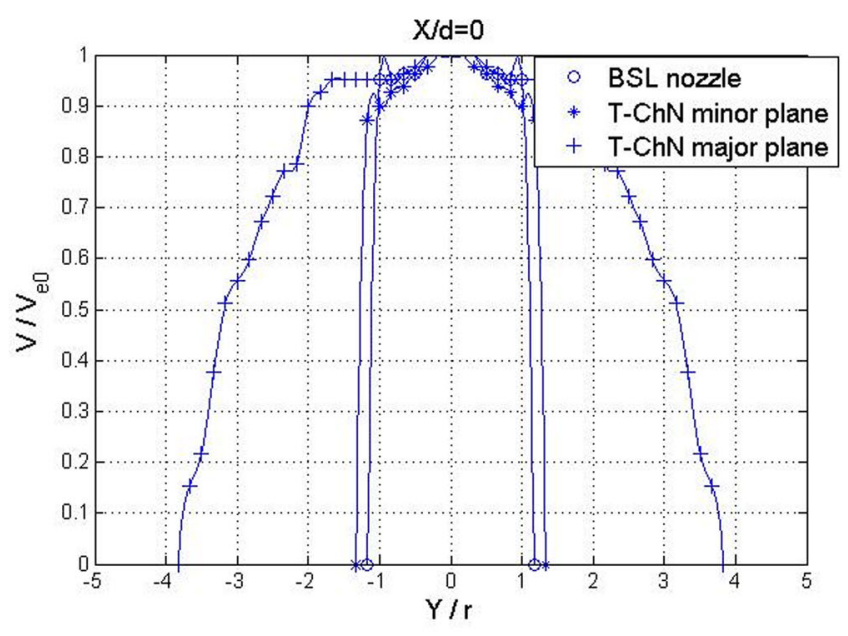

(a)

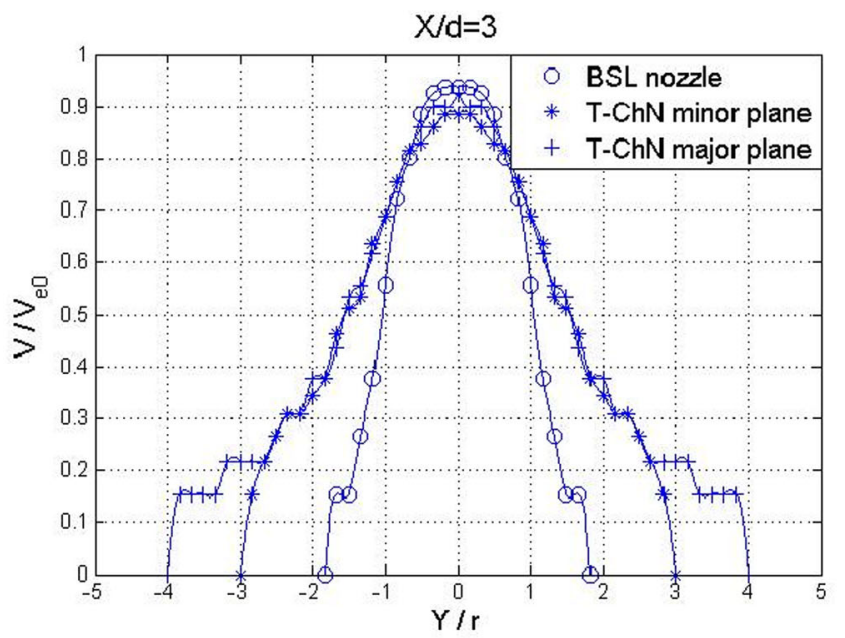

(c)

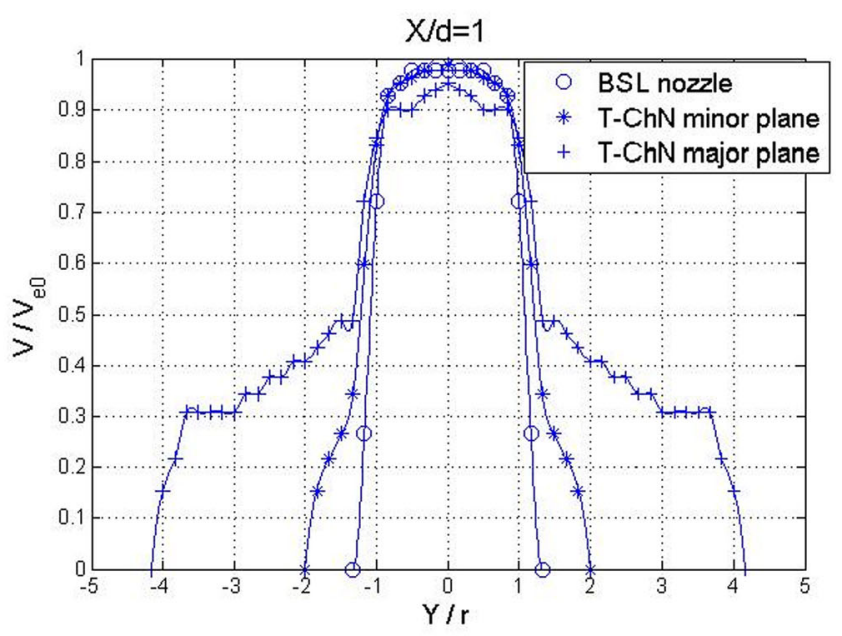

(b)

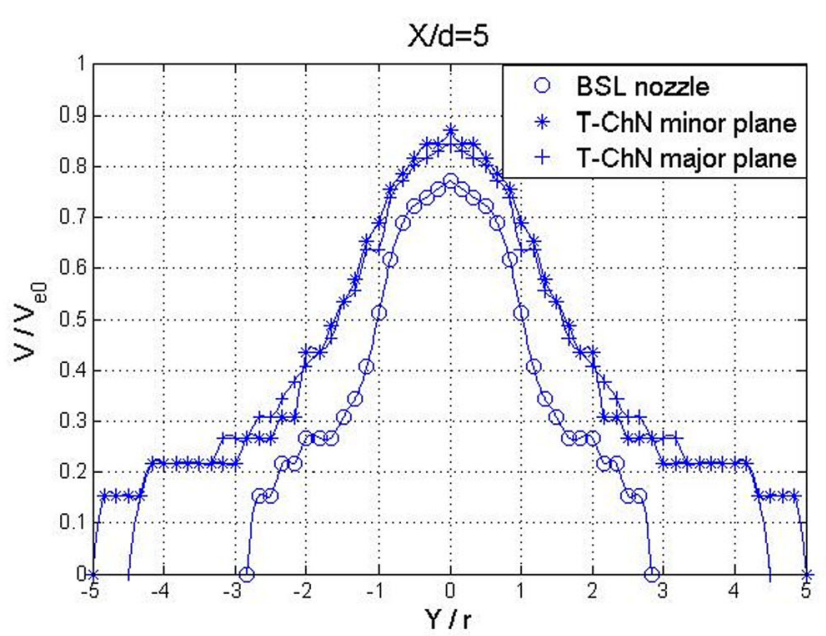

(d)

Figure 8. Mean velocity profiles in radial direction for a Reynolds number of 7400: for tabbed chevron nozzle and baseline nozzle.

which $\frac{V_{e 0}}{V_{x 0}}$ remains constant. The decay constant is given by the inverse of slope of the linearly increasing portion of the graph in figure 4.

One of the key parameters that characterizes the jet spread is the jet half-width $\left(Y_{0.5}\right)$, which is defined as the radial distance from the jet axis to the point where the velocity is half of the centreline value [15]. The spread rate of the jet is given as the slope of the linearly increasing portion of the $\frac{Y_{0.5}}{d}$ vs $\frac{X}{d}$ graph [27]. The comparison provided in table 2 shows that the values from the present study match reasonably well with those reported in literature. Plots of the radial velocity profiles in figure 5 show that the measurements made in this study (for BSL nozzle) conform to the development of the self-similar trend in free jets [27] - the circular jet exhibits self-similarity after $X / d=8$.

\subsection{Studies on jet mixing with chevrons}

3.2a Quantitative studies: In this section, the mixing enhancement accomplished by the use of the tabbed chevron nozzle is examined by way of jet profile development in the near-field of the nozzle. The extent of mixing is characterized mainly by the axial and radial profiles of mean velocity. The comparison focusses on how mixing progresses for free jets issuing from the three nozzles: (i) the baseline circular nozzle, (ii) the conventional chevron nozzle and (iii) the improvised tabbed chevron nozzle.

Figure 6 represents the centreline decay plots for the 3 nozzles (error bars are indicated in the plot only for the baseline nozzle (BSL nozzle) for the sake of clarity). It is seen that the tabbed chevron nozzle (T-ChN) has the lowest potential core length as well as the fastest decay of centreline velocity in the near-field (up to $5 d$ ). Beyond this 


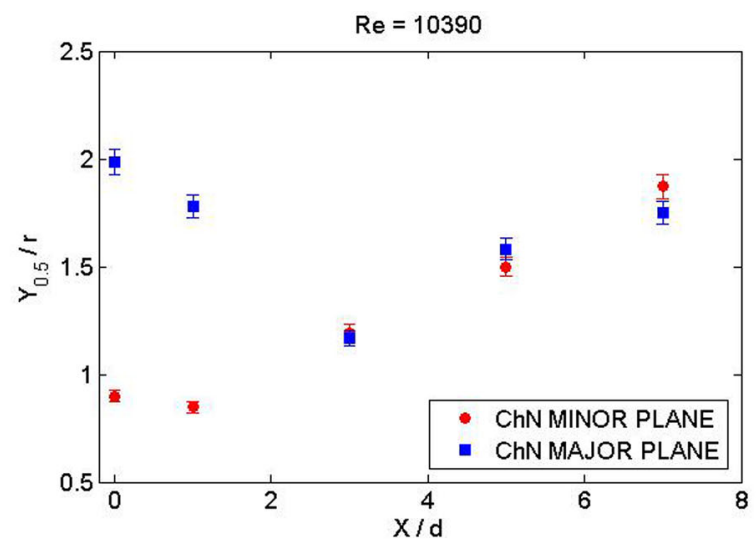

(a)

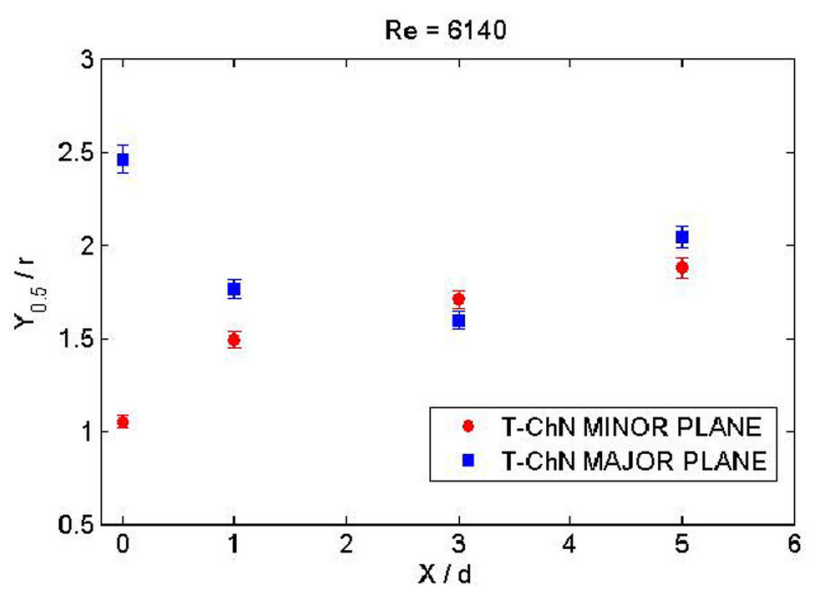

(b)

Figure 9. Development of jet half-width: (a) 6-tooth conventional chevron and (b) 6-tooth tabbed chevron nozzle.

distance, no significant velocity difference was observed for the 3 nozzles. This trend was observed for the other Reynolds numbers as well. For the case of lobed nozzles, a similar observation has been made by Nastase and Meslem
[13]. This can be attributed to the axial decay of the strength of the streamwise vortices. The tabbed-chevron nozzle has the lowest potential core length among all the nozzles (see table 3), which is also indicative of the higher rate of momentum-mixing.

The streamwise development of radial profiles of mean velocity for the conventional chevron nozzle is compared to that of the circular nozzle in figure 7. For chevron nozzle, the velocity profiles are shown along (i) the major plane and (ii) the minor plane. The following observations can be made from the plots. (i) In the near-field of the nozzle exit, the velocity profiles along the minor and major planes are significantly different from each other and jet spread along the major plane is higher than that along the minor plane. (ii) Moving downstream, velocity profiles in the two planes progressively become similar and at $X / d=3$ the difference is minimal. (iii) As previously observed in the case of coflowing jets [15], the circular nozzle results in a higher velocity in the proximity of the centreline $(-r<Y<r)$ in comparison with the chevron nozzle. Similarly, the chevron nozzle results in a relatively higher velocity in the outer region $(Y>r)$ in comparison with circular nozzle. The reason for this can be inferred as follows. Chevrons induce an increased rate of transfer of momentum from the core zone to the outer zone (which originally has low momentum). This redistribution results in momentum levels that are different from those for circular nozzle in the respective zones. In figure $7 b$ and $c$, this difference can be observed in the velocity profiles for the two nozzles. Hence, in singleflow jets, chevrons cause a lateral momentum transfer from the core jet to ambient. However, this mechanism of transverse momentum exchange is prominent only close to the jet exit.

Figure 8 shows the development of velocity profiles in the radial plane for the tabbed chevron nozzle; the mechanism of transverse momentum exchange from the highvelocity jet to the low-velocity ambient (discussed in the previous paragraph) can be seen in figure $8 \mathrm{c}$. In this case also, the velocity profiles in the major and minor planes are

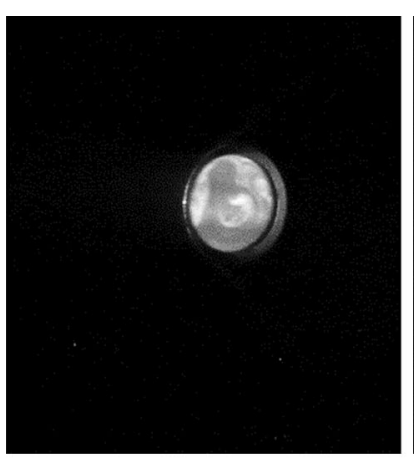

(a)

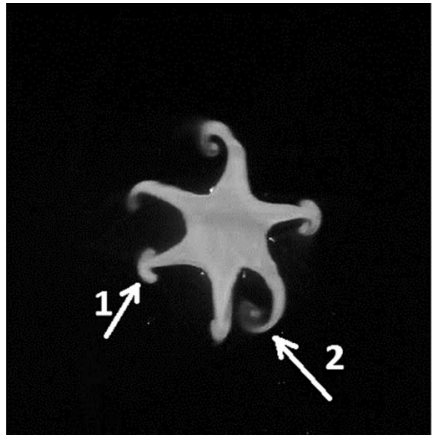

(b)

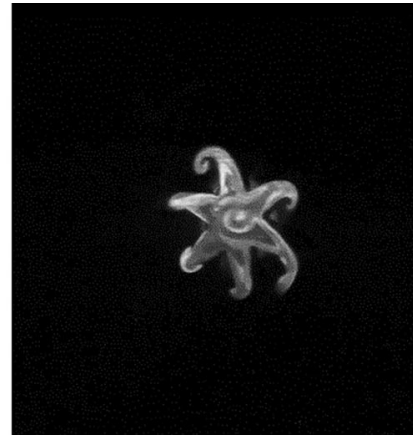

(c)

Figure 10. Jet cross section at the exit for (a) BSL nozzle, (b) ChN nozzle and (c) T-ChN nozzle. 


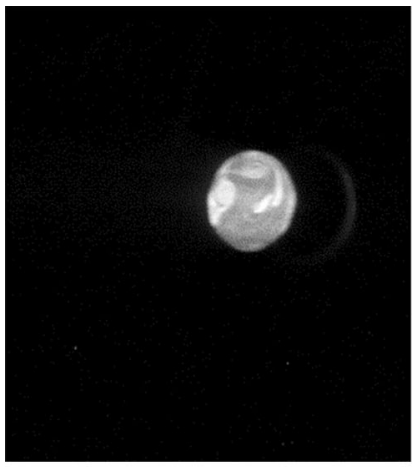

(a)

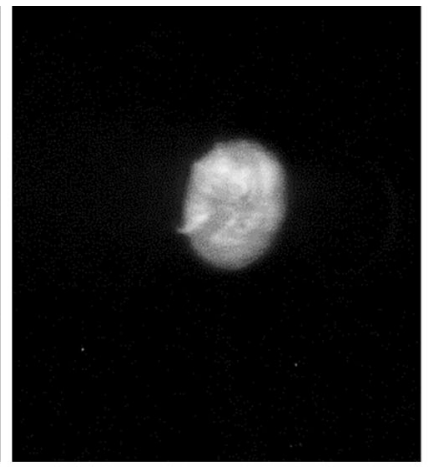

(b)

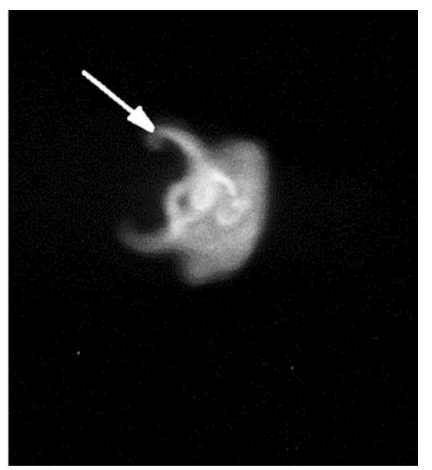

(c)

Figure 11. Evolution of jet cross section for the circular nozzle at (a) $X / d=1$, (b) $X / d=2$ and (c) $X / d=3$ (arrow in (c) indicates the development of an azimuthal structure).

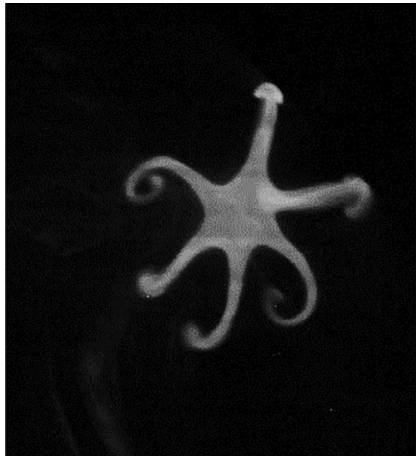

(a)

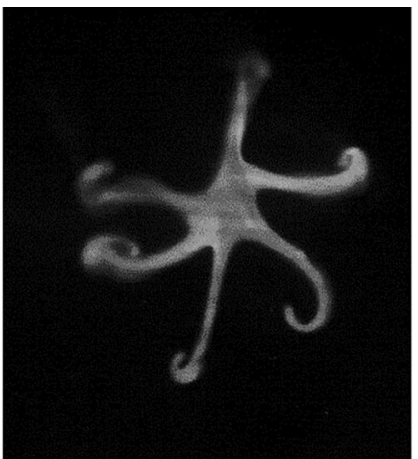

(b)

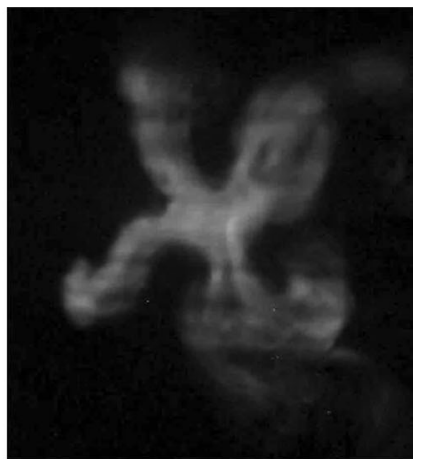

(c)

Figure 12. Progressive evolution of the jet cross section at (a) $X / d=1, X / d=2$ and $X / d=3$.

drastically different close to the nozzle exit (with the major plane showing increased jet spread), further downstream at $X / d=3$ the velocity profiles of both the major plane and the minor plane are identical to each other.

Figure 9a shows the variation of jet half-width for conventional chevron nozzles. A noticeable difference in the half-width evolution of the major and minor planes can be observed: The width in the major plane decreases initially and then increases, while the width along the minor plane increases almost continuously (although at lower Reynold numbers there is a slight decrease from $0 d$ to $1 d$ followed by a continuous increase). Similar observations can be made on the comparison of growths of jet width for the tabbed chevron nozzle (figure 9b). A significant increase in jet width, as expected, can be seen for the tabbed nozzle from that for the conventional chevron nozzle. Also, interestingly the trend for the evolution of half-widths for chevron jet is very similar to that reported for jets from lobed nozzles by Nastase and Meslem [13].

3.2b Flow visualization studies: Figure 10 shows the jet cross section at the jet exit for the nozzles considered in the present study, the star-like structure that characterize nearfield of the conventional and tabbed chevron nozzles may be noted. Two different types of streamwise structures are noticeable for the $\mathrm{ChN}$ nozzle in figure 10b (the mushroom-shaped structure (arrow-1) and the rolled vortex type structure (arrow-2)). Further evolution of the jets for the three configurations considered here (circular, conventional chevron and tabbed chevron) is discussed below.

3.2c Circular nozzle: From figure 11 it can be seen that for the circular nozzle the jet cross section remains circular till $X / d=2$. Subsequently, at $X / d=3$ the emergence of azimuthal structures can be noted.

3.2d Conventional chevron nozzle: At $X / d=1$ (figure 12(a)) the evolution of the streamwise structures is observed. The vorticity associated with the lobes of the jet cross section deforms the "fingers" of the jet cross section (figure 12(a)). Roll-up of the vortices continues as the jet moves downstream (figure 12(b)). Further downstream at $X / d=3$ (figure $12(\mathrm{c})$ ), the jet no longer retains its characteristic 6-lobed structure and the adjacent legs of the jet 


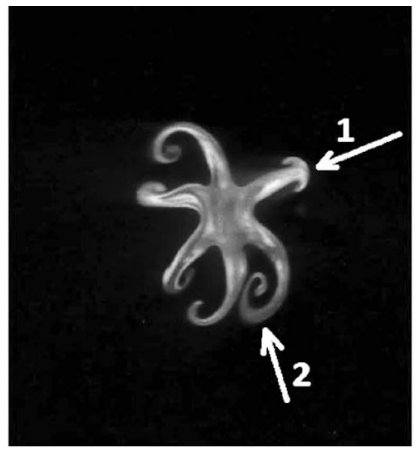

(a)

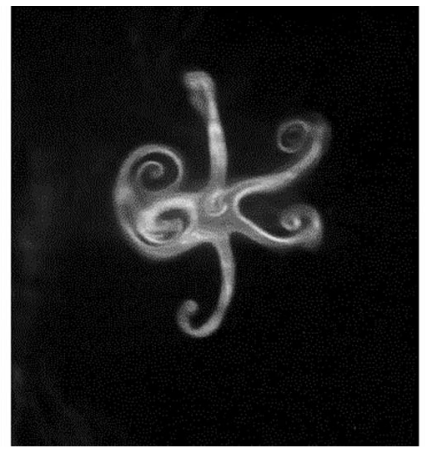

(b)

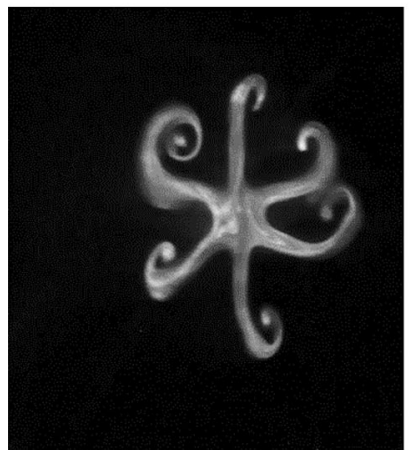

(c)

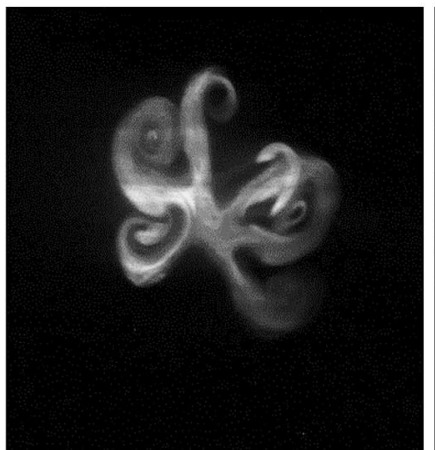

(d)

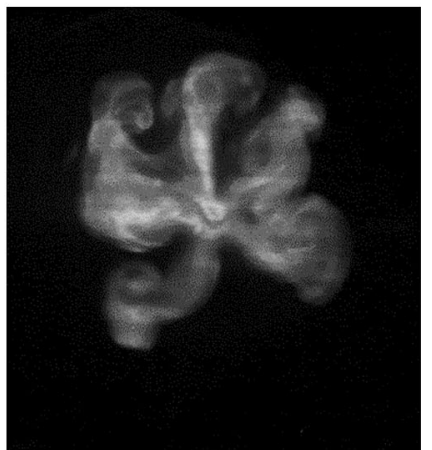

(e)

Figure 13. Progressive evolution of the jet cross section at (a) $X / d=1$, (b) $X / d=2$, (c) $X / d=3$, (d) $X / d=4$ and (e) $X / d=5$.

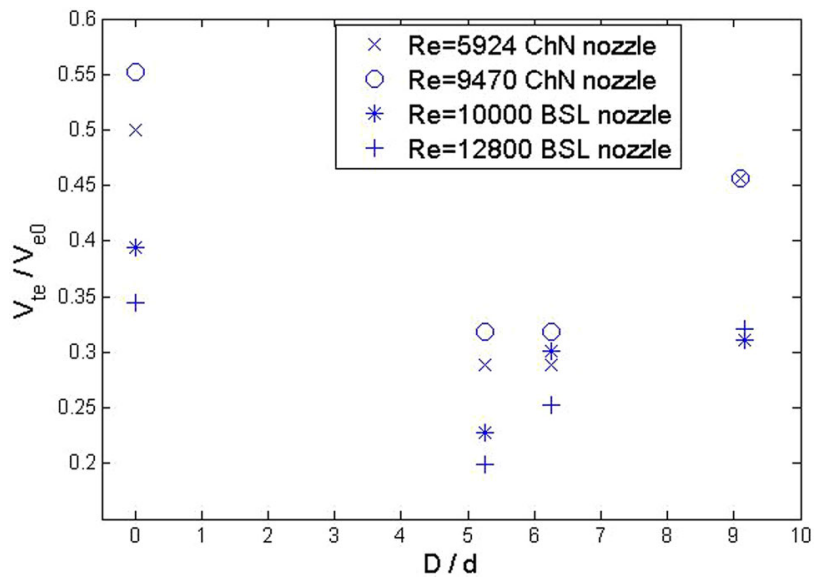

Figure 14. Non-dimensional centreline velocity at tube exit for different diameter ratios and $L / d=14$.

cross section progressively merge with each other. No streamwise structures were visible further downstream.

3.2e Tabbed chevron nozzle: The near-field evolution of the jet issuing from the newly devised tabbed chevron nozzle is shown in figures $13 \mathrm{a}-\mathrm{e}$. It may be recalled from section 2.1 that the introduction of tabs into the chevron geometry was made with the intention of enhancing the rate of mixing. The flow visualization images show that while the vortex pattern is similar to that with the conventional chevron nozzle (figure 12), there is a prominent increase in the apparent intensity of the streamwise vortices. The jet cross section at $X / d=1$ shows the existence of mushroom-shaped streamwise structure (arrow-1) and rolled vortex type streamwise structure (arrow-2). It can be seen that the jet cross section is extensively deformed as it proceeds downstream due to intense vorticity associated with the jet. At $X / d=2$ (figure 13(b)), two adjacent legs of the jet tend to cross over, which probably characterize stronger vorticity. At $X / d=5$, adjacent vortices have merged and the jet loses its original shape, though the streamwise structures are seen to be present. The flow visualization images show that the streamwise vortices endure farther downstream and are more intense for the tabbed chevron nozzles. It follows from the work of Liepmann and Gharib [4] that such structures increase the rate at which fluid is entrained into the jet. However, more studies are required to quantify this effect.

The flow visualization results show that the tabbed chevron geometry results in persistence of streamwise vortices over a longer axial distance from the nozzle exit compared with a conventional chevron; the quantitative measurements discussed in section 3.2 are also supportive of this observation (because of persistence of streamwise 


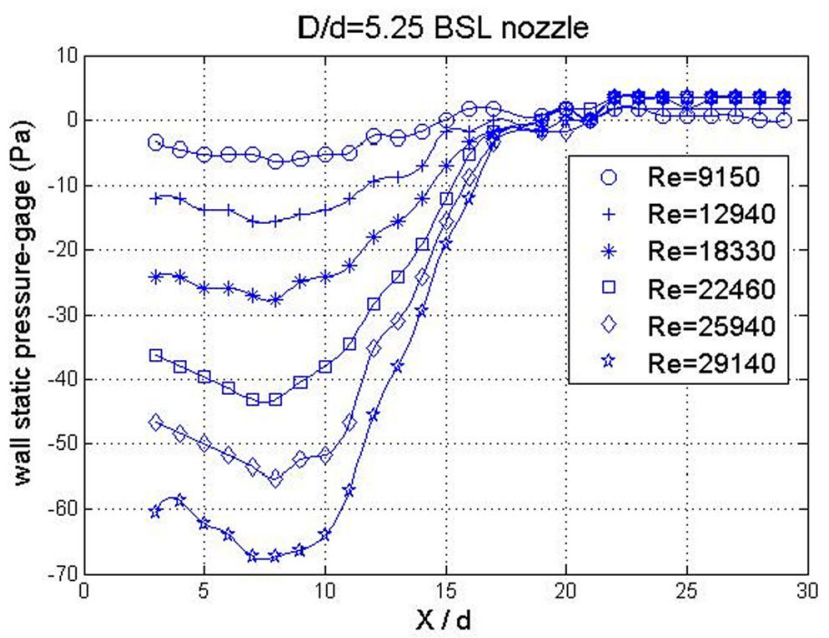

(a)

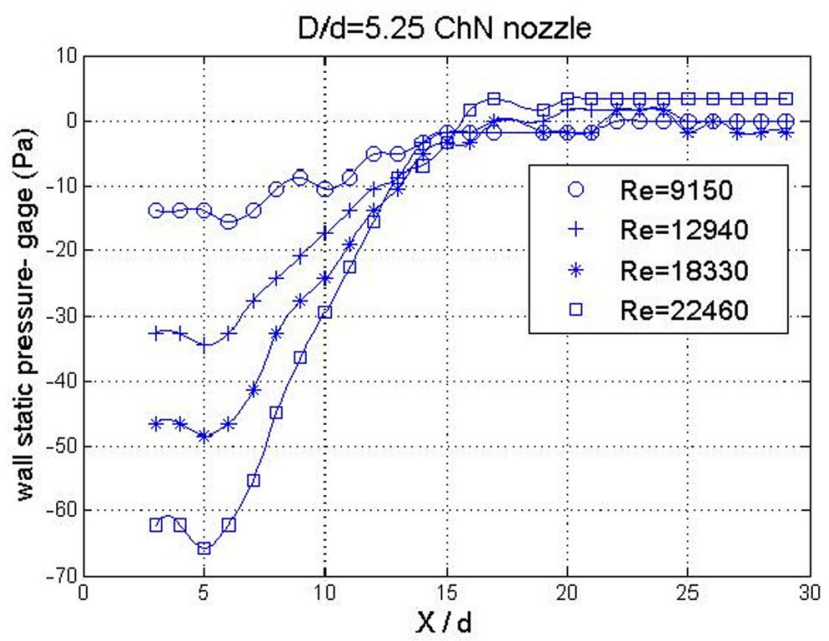

(c)

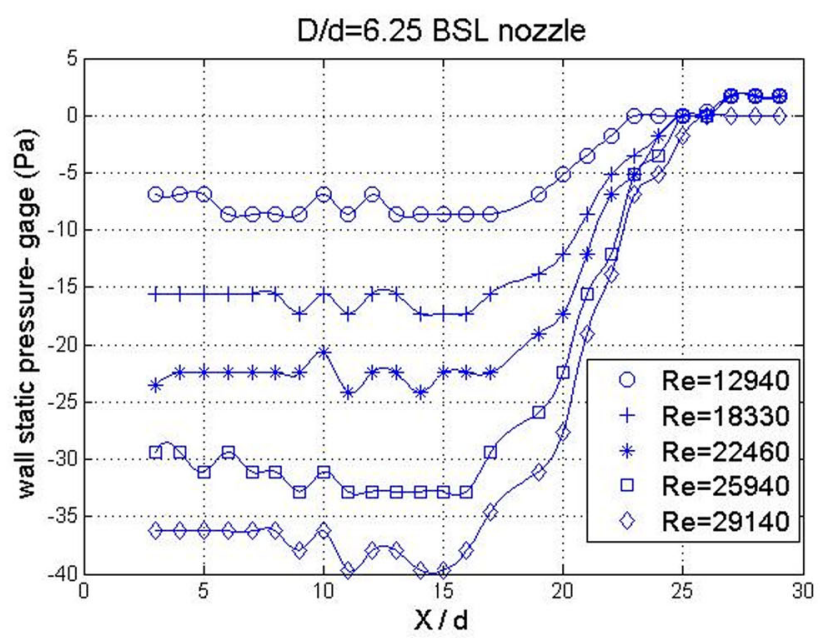

(b)

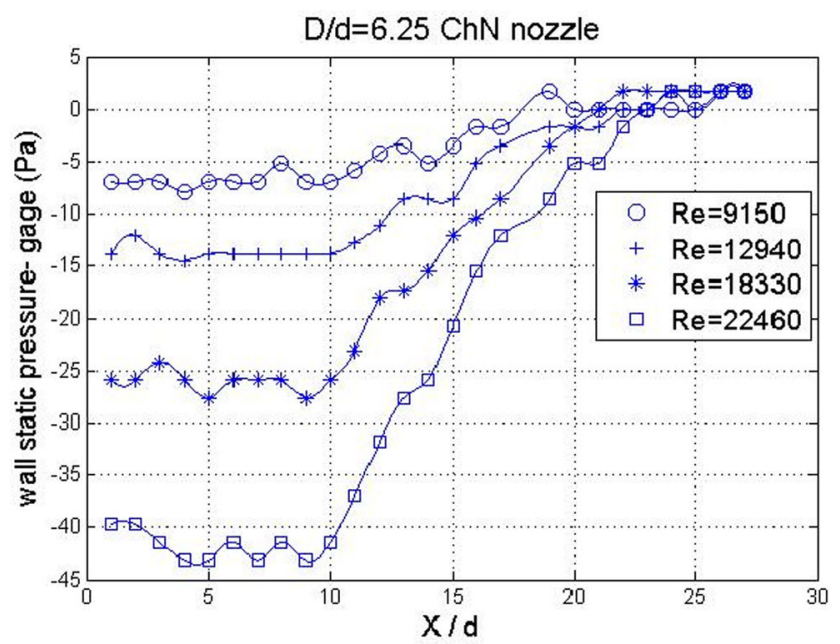

(d)

Figure 15. Variation of wall static pressure with axial distance, for BSL and ChN nozzles for a tube with $L / d=29$.

structures for longer distances, the tab chevron shows a reduced potential core length and faster centreline decay in the near-field- $X / d<5$ ). Beyond $X / d=5$ all the three configurations tested had almost identical centreline velocities; this is due to the fact that the streamwise vortices of the $\mathrm{ChN}$ and T-ChN nozzles completely diminish within $\mathrm{X} /$ $d=5$; hence, their effect on mixing and entrainment is prominent only close to the exit. For the conventional chevron nozzle in the transonic regime, Alkislar et al [30] have reported that enhanced mixing is constrained to the near field of the nozzle.

\subsection{Studies on confinement of jets from chevron nozzle}

While there are experimental studies reported for confined jet mixing with circular and lobed nozzles, no data are available for jets issuing from chevron nozzles into a mixing tube. The present study examines development of the jet profile in the presence of a confining cylindrical tube attached as shown schematically in figure $1(\mathrm{~b})$. The plots of centreline velocity at tube exit for different Reynolds numbers (figure 14) show that the effect of confinement is to reduce the jet velocity along the axis. This can be inferred from the fact that for a given nozzle and a given Reynolds number, the tube exit centreline velocities for confined jet (which have non-zero value of $D / d$ ) are always lower than that for the unconfined jet $(D / d=0)$ (see figure 14). Also, it can be seen that the centreline velocity at tube exit decreases as confinement increases (in figure 14 it can be seen that for the confined jet case ( $D / d$ non-zero) the centreline velocity decreases with a decrease in $D / d$ ). This observation is in line with that made by Risso and Fabre [21] for circular jets with confinement. (It may be noted that the plots are not aimed at a comparison of values for 


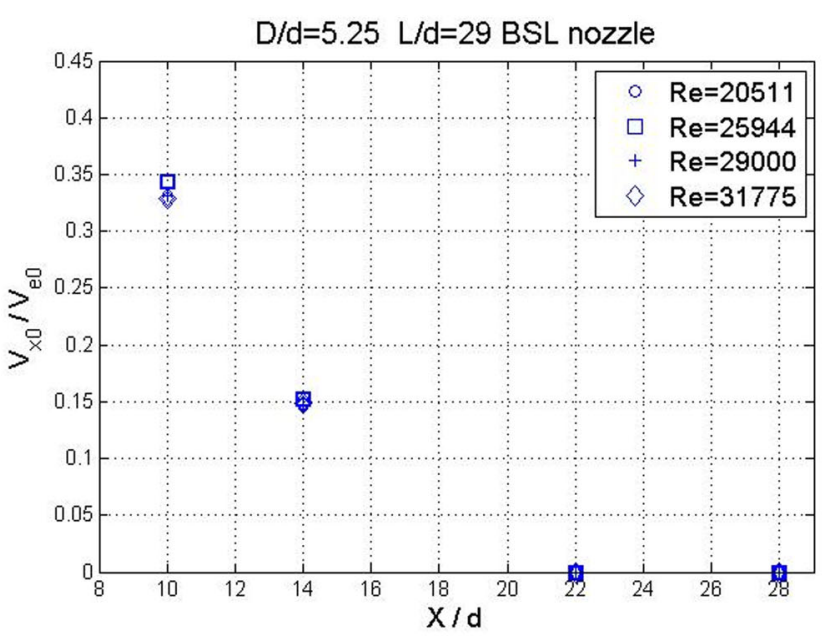

(a)

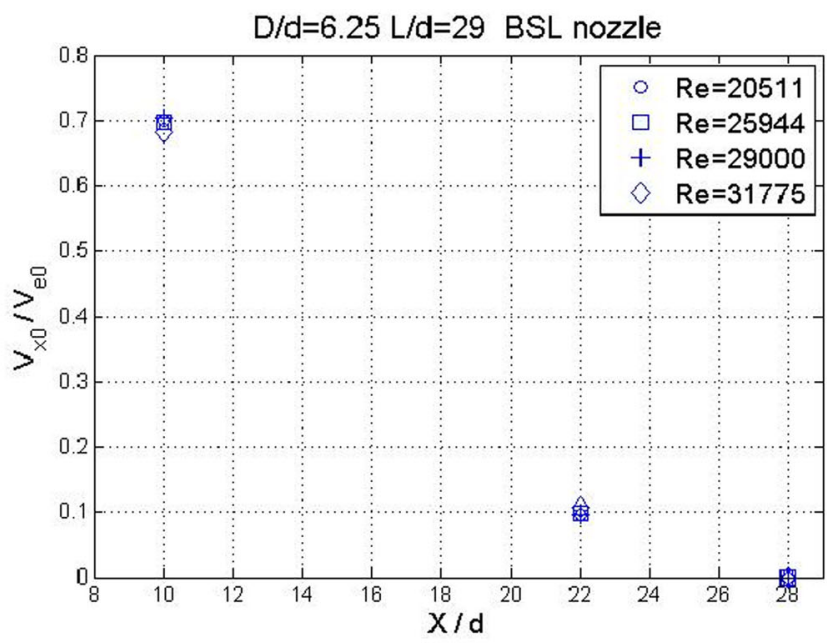

(b)

Figure 16. Variation of dimensionless tube centreline velocity with $X / d$ for the BSL nozzle and with $L / d=29$.

Table 4. Values of $L_{b}$ and $L_{a d v}$ for different diameter ratios.

\begin{tabular}{|c|c|c|c|c|}
\hline \multirow[b]{2}{*}{ Tube diameter ratio $D / d$} & \multicolumn{2}{|c|}{$L_{a d v} / d$} & \multicolumn{2}{|c|}{$L_{b} / d$} \\
\hline & BSL nozzle & $\mathrm{ChN}$ nozzle & BSL nozzle & ChN nozzle \\
\hline 5.25 & 8 & 5 & 22 & 19 \\
\hline 6.25 & 15 & 10 & 25 & 22 \\
\hline
\end{tabular}

baseline and chevron nozzles: data are presented over a range of Reynolds numbers, in order to reflect the trend of variation of centreline velocity with mixing tube diameter.) Figure 15 shows that the wall static pressure for the confined jet initially remains constant and then sharply increases and levels off to a constant value. Interestingly, the axial location where the pressure starts to rise and the location where it levels off remain the same irrespective of Reynolds number. The increase in wall static pressure causes an adverse pressure gradient, which, in combination with wall friction, is the primary cause for the jet break-up in confined turbulent jets.

Liberzon and Fernando [23] have shown that the static pressure attaining a constant value is indicative of the occurrence of jet break-up. Subsequently, the jet has very low velocity. The observations made in [23] were for a tube with the upstream end open and the downstream end closed. Results of the present study show that a similar interaction of the pressure field with the confinement takes place for tubes with open downstream end and closed upstream end; this is discussed in the following paragraph.

For the tube with $D / d=5.25$, at $X / d=22$ and 28 we can see that the static pressure has levelled off to a constant value (figure 15a) and the dimensionless centreline velocity for those locations is negligibly small (figure 16a). Hence the jet has broken up into diffusive turbulence [22]. However, for $X / d$ values of 10 and 14 where the static pressure does not level off, it can be seen that there is a finite centreline velocity. The same conclusions can be drawn for the tube with $D / d=6.25$ (figures $15 \mathrm{~b}$ and $16 b)$. Hence, from the results presented here it can be concluded that the axial location where the jet break-up has occurred is given by the location where the static pressure becomes a constant. These results provide support for extending the observations on break-down made for tubes with "closed-downstream ends" to the present configuration (figure 1b).

From the static-pressure plots of figure 15, two lengths that characterize the confined jet can be defined: (i) $L_{a d v}$, the axial location from jet exit where the pressure starts to rise (signifies the length at which the confining effect of the tube is felt by the jet) and (ii) $L_{b}$, the axial distance from jet exit to the location where the jet breaks up (indicated by the static pressure tending to be constant).

From table 4 it is clear that the location where the confining effect of the tube is felt is much upstream for the tube with smaller $D / d$ ratio and for the confined jet with chevron nozzle (in comparison with the circular nozzle). The same observation holds good for the break-down length as well. 
The impact of the diameter of the confining tube (represented by $D / d$ ) on the jet can be explained as follows: the jet, as it issues from the exit of the nozzle, expands to hit the wall of the confining tube and the presence of the wall imposes an adverse pressure gradient. The concomitant increase in static pressure, combined with the effect of viscosity near the wall, contributes to break-down of the jet [23]. When the tube diameter is relatively small (lower values of $D / d$ ), the jet comes in contact with the wall much faster (at lower $L / d$ ); the wall-effect is felt over a longer distance and hence the break-down takes place closer to the nozzle exit, leading to a lower centreline velocity at exit of the tube.

Also, since the chevron jet has a higher jet spread compared with the circular nozzle, it comes in close proximity with the tube walls much earlier than the circular jets. Hence the adverse pressure gradient is felt over a longer distance along the axis and this, in turn, leads to rapid break-down and completely diffusive turbulence.

\section{Conclusions}

The present investigation was undertaken to study mixing enhancement of low-Reynolds-number jets in the presence of chevrons and to analyse the impact of confinement on the rate of mixing of single jets issuing from chevron nozzle. One of the key objectives was to further enhance the mixing performance of chevrons by way of a simple geometry modification. The mixing performance of an improvised chevron nozzle (with tabs provided at the exit) was compared to that of a conventional nozzle and a baseline circular nozzle, based on mean velocity profiles. The tabbed geometry was found to promote the formation and sustenance of vortex structures, which can eventually result in enhanced entrainment. Development of the chevron jet in the near field was also qualitatively examined by way of flow visualization studies. Two types of streamwise structures were observed for the chevron nozzle. The streamwise vorticity associated with the chevron jet was found to deform the jet cross section significantly. Persistence of streamwise structures for longer distances from jet exit was found to be an important factor in governing its mixing characteristics.

The effect of confinement on jets issuing from conventional chevron nozzles was studied for an open-end configuration. It was observed that presence of the confining tube leads to a faster decay in centreline velocity and complete break-down of the jet into diffusive turbulence. The jet break-down could be attributed to the adverse pressure gradient developed in the presence of confinement. Jet break-down occurs faster with mixing tubes of smaller diameters. In comparison with the circular nozzle, the chevron nozzle results in relatively faster jet break-down, due to the enhanced spreading rate of the jets. Findings of the present study contribute directly to the application of chevrons for mixing enhancement at low speeds.

$\begin{array}{ll}\text { List of symbols } \\ A & \text { jet decay constant } \\ b & \text { spread rate of the jet } \\ D & \text { diameter of the mixing tube }(\mathrm{mm}) \\ d & \text { diameter of the nozzle exit }(\mathrm{mm}) \\ L & \text { length of the mixing tube }(\mathrm{mm}) \\ \mathrm{Re} & \text { Reynolds number } \\ r & \text { radius of nozzle }(\mathrm{mm}) \\ V & \text { velocity (m/s) } \\ V_{e 0} & \text { centreline velocity at the jet exit }(\mathrm{m} / \mathrm{s}) \\ V_{t e} & \text { centreline velocity at exit of the mixing tube }(\mathrm{m} / \mathrm{s}) \\ V_{x 0} & \text { centreline velocity at any given } X \text { location }(\mathrm{m} / \mathrm{s}) \\ X & \begin{array}{l}\text { distance from nozzle exit, measured along } \\ \text { streamwise direction (mm) }\end{array} \\ Y & \begin{array}{l}\text { distance from the nozzle exit measured along the } \\ \text { radial (transverse) direction (mm) }\end{array} \\ Y_{0.5} & \text { jet half-width (mm) }\end{array}$

\section{References}

[1] Hu H, Saga T, Kobayashi T and Taniguchi N 2001 A study on a lobed jet mixing flow by using stereoscopic particle image velocimetry technique. Phys. Fluids 13: 3425-3441

[2] Brodkey R 1975 Turbulence in mixing operations. New York: Academic Press

[3] Bernal L P and Roshko A 1986 Streamwise vortex structures in plane mixing layers. J. Fluid Mech. 170: 499-525

[4] Liepmann D and Gharib M 1992 The role of streamwise vorticity in the near field entrainment of round jets. J. Fluid Mech. 245: 643-668

[5] Zaman K B M Q, Reeder M F and Samimy M 1994 Control of an axisymmetric jet using vortex generators. Phys. Fluids 6: 778-793

[6] Reeder M F and Samimy M 1996 The evolution of a jet with vortex generating tabs: real-time visualization and quantitative measurements. J. Fluid Mech. 311: 73-118

[7] Verma S B and Rathakrishnan E 2003 Influence of aspect ratio on the mixing and acoustic characteristics of plain and modified elliptic slot jets. Aerosp. Sci. Technol. 7: 451-464

[8] Arun Kumar P and Rathakrishnan E 2013 Truncated triangular tabs for supersonic jet control. J. Prop. Power 29: $50-65$

[9] Srikrishnan A R, Kurian J and Sriramulu V 1996 Enhancement of thermal mixing in coaxial supersonic jets. J. Prop. Power 12: 730-735

[10] Srikrishnan A R, Kurian J and Sriramulu V 1996 An experimental investigation of thermal mixing and combustion in supersonic flows. Combust. Flame 107: 464-474

[11] Nastase I, Meslem A and Gervais P 2008 Primary and secondary vortical structures contribution in the entrainment of low Reynolds number jet flows. Exp. Fluids 44: 1027-1033 
[12] Nastase I and Meslem A 2008 Vortex dynamics and entrainment mechanisms in low Reynolds number orifice jets. J. Visualization 11: 309-318

[13] Nastase I and Meslem A 2010 Vortex dynamics and mass entrainment in turbulent lobed jets with and without lobe deflection angles. Exp. Fluids 48: 693-714

[14] El Hassan M and Meslem A 2010 Time resolved stereoscopic particle image velocimetry investigation of the entrainment in the near field of circular and daisy shaped orifice jets. Phys. Fluids 22: 035107

[15] Callender B, Gutmark E J and Martens S 2010 Flow field characterization of co-axial conical and serrated (chevron) nozzles. Exp. Fluids 48: 637-649

[16] Zaman K B M Q, Bridges J E and Huff D L 2010 Evolution from 'tabs' to 'chevron technology' - a review. In: Proceedings of the 13th ASIAN congress of Fluid Mechanics, Dhaka, Bangladesh, 17-21 December 2010

[17] Bridges J and Brown C A 2004 Parametric testing of chevrons on single flow hot jets. NASA Technical Memorandum No. NASA TM-213107

[18] Kennedy J and Fitzpatrick J 2010 The effect of chevrons on the turbulence characteristics of jets. In: Proceedings of the 16th AIAA/CEAS Aeroacoustics Conference, Stockholm, Sweden, 7-9 June 2010

[19] Mihaescu M and Gutmark E 2007 Modelling of the flow and acoustical field due to a single jet with chevrons. In: Proceedings of the 43rd AIAA/ASME/SAE/ASEE Joint Propulsion Conference and Exhibit, Cincinnati, Ohio, 8-11 July 2007

[20] Opalski A B, Wernet M P and Bridges J E 2005 Chevron nozzle performance characterization using stereoscopic
DPIV. In: Proceedings of the 43rd AIAA Aerospace Sciences Meeting and Exhibit, Reno, Nevada, 10-13 January 2005

[21] Risso F and Fabre J 1997 Diffusive turbulence in a confined jet experiment. J. Fluid Mech. 337: 233-261

[22] Voropayev S I, Sanchez X, Nath C, Webb S and Fernando H J S 2011 Evolution of a confined turbulent jet in a long cylindrical cavity: homogeneous fluids. Phys. Fluids 23: 115106

[23] Liberzon D and Fernando H J S 2014 Pressure distribution in confined jet flow. J. Fluids Eng. Trans. ASME 136: 031202-1

[24] Singh G, Sundararajan T and Bhaskaran K A 2003 Mixing and entrainment characteristics of circular and noncircular confined jets. J. Fluids Eng. Trans. ASME 125: 835-842

[25] Phanindra B C and Rathakrishnan E 2010 Corrugated tabs for supersonic jet control. AIAA J. 48: 453-465

[26] Viswanath K B S N and Ganesan V 2001 Experimental and numerical investigation of an axisymmetric free jet. Indian J. Eng. Mater. Sci. 8: 189-197

[27] Hussein H J, Capp S and George W K 1994 Velocity measurements in a high-Reynolds number, momentum conserving, axisymmetric, turbulent jet. J. Fluid Mech. 258: 31-75

[28] Abdel-Rahman A A, Al-Fahed S F and Chakroun W 1996 The near field characteristics of circular jets at low Reynolds numbers. Mech. Res. Commun. 23: 313-324

[29] Panchapakesan N R and Lumley J L 1993 Turbulence measurements in axisymmetric jets of air and helium, part 1: air jet. J. Fluid Mech. 246: 197-223

[30] Alkislar M B, Krothapalli A and Butler G W 2007 The effect of streamwise vortices on the aeroacoustics of a Mach 0.9 jet. J. Fluid Mech. 578: 139-169 